\title{
DEBATES
}

\section{Confianza, capital social y democracia: una compleja articulación para el desarrollo}

\section{Trust, social capital and democracy: a complex joint for development}

\section{Francisco Ganga Contreras \\ Aldo Cassinelli Capurro}

\section{Resumen}

Últimamente se ha visualizado el progreso en Latinoamérica, principalmente desde una perspectiva economicista. Hoy, se están realizando indagaciones orientadas a sostener el crecimiento, pero apuntando al desarrollo, lo cual puede ser convertido en capital social. La democracia se transforma en un factor clave en este desafío y con ello, la confianza en los individuos y instituciones. En este sentido, el propósito central de este trabajo es analizar, los aspectos más relevantes relacionados con la triada confianza, capital social y democracia y su impacto en el desarrollo. Para alcanzar estos fines, se recurrió primordialmente a fuentes secundarias de información, lo cual implicó revisión de artículos que abordan esta problemática. Se concluye que una sociedad que aspire al desarrollo, debe avanzar en alcanzar una vertebración institucional que posibilite la resolución adecuada de las problemáticas y demandas que tiene la sociedad, de tal forma de lograr que ésta retribuya con apropiados niveles de confianza.

\section{Palabras clave}

Latinoamérica; Democracia; Instituciones; Capital Social; Confianza; Desarrollo; Desarrollo Humano.

\begin{abstract}
Lately, it has been seen progress in Latin America, mainly from an economic perspective. Currently, it has been conducted research aimed at sustaining the growth, but focused on the country's development, which can be converted into social capital. Democracy becomes a key factor on this challenge and thus confidence in individuals and institutions. In this sense, the central purpose of this paper is to analyze the most relevant aspects of trust, social capital and its impact on democracy and development. To achieve these objectives, it is primarily used secondary sources of information, which involved review of articles addressing this issue. The conclusion is that a society that aspires the development should coordinate institutions to solve the society's problems and demands, so that society responds with appropriate confidence levels.
\end{abstract}

\section{Keywords}

Latin America; Democracy; Institutions; Social Capital; Trust; Development; Human Development. 


\section{Introducción}

La discusión sobre el desarrollo ha tenido diversas corrientes de aproximación, por una parte existen las netamente economicistas, aquellas que nos sugieren que éste se alcanza al superar un umbral de ingresos y es ello lo que marca la pauta para que dicha sociedad sea reconocida como avanzada. El ingreso logrado importa contar con una serie de acuerdos básicos que la población asume como óptimos y desde ese punto de vista se da por completado un ciclo de desarrollo.

En otra vereda se encuentran aquellos que asumen que el desarrollo no sólo está asociado al nivel de ingresos, sino más bien a la construcción de cierto entramado institucional que permite a su población ser capaz de desplegar su potencialidad en beneficio propio, pero también en beneficio de la sociedad en la cual está inserto.

En ambos casos parece existir un punto en común, cual es la creación de valor social y es este valor el que nos interesa analizar de manera detallada, teniendo como aproximación conceptual la construcción de capital social por parte del colectivo nacional. Por cierto, como ocurre reiteradamente en las ciencias sociales no hay una variable que explique la totalidad de un fenómeno, menos uno tan complejo como el desarrollo o en este caso el capital social, por ello se realizarán aproximaciones sucesivas que nos permitan ir avanzando en una explicación racional y confiable.

Existe una abundante producción académica que vincula un complejo entrecruzamiento de variables tales como confianza social, confianza en las instituciones y satisfacción con la democracia. El punto común es entender que la confianza se sitúa como nodo articulador de los procesos políticos-sociales. A ellas se tiende a agregar, dependiendo de la región en estudio, variables como la distribución del ingreso entendiéndola como aquella que tiende a afectar la equidad interna de los países, así como otros indicadores de corte económico para tratar de distinguir la relación que existe entre ellas y el desarrollo de las sociedades en base a la generación de riqueza, todo lo cual queda bajo la responsabilidad de la meta organización denominada Estado quien se erige como responsable de un modelo que asegure un orden social básico que se traduce en gobernabilidad. Esta compleja integración de variables, que claramente no son las únicas que participan, ayudarán a concebir la manera que afectan a la estabilidad del sistema democrático mediante lo que se ha dado en denominar los arreglos institucionales a través de los que se implementa la acción de los miembros de la sociedad estén o no organizados.

Por cierto, sise entiende la confianza como aquella fiabilidad mutua que se genera entre individuos $\mathrm{u}$ organizaciones mediante la existencia de contenidos cognitivos que se vinculan, por una parte a las estrategias de acción y por otra a las expectativas que cada cual tiene del otro, nos permitiría asumir un abanico de 
posibilidades respecto de los resultados de dichas interacciones en el plano social. Dicho resultado serían las certezas que les posibilitan a los individuos tomar decisiones de determinada manera basado en una realidad existente y validada socialmente.

Lo anterior nos lleva a situarnos en el plano de identificar una conceptualización mínima del denominado Capital Social, el cual se compone de múltiples variables aquí recogidas, pero que se expresa a través de la interacción entre individuos/instituciones y que tiene entre sus elementos predominantes a la confianza.

Es por ello que los estudio, basado en la confianza, y las diferentes líneas que posee son fundamentales para comprender la construcción de relaciones que se generan en la sociedad y de manera directa en la valoración que se tiene de la democracia representativa como constructo ideológico, el cual norma dichas relaciones mediante el diseńo de instituciones que enmarcan las acciones de los individuos, ya que en muchos casos se da por sentada su existencia y funcionamiento sin cuestionar necesariamente la eficacia de las mismas o su pertinencia para determinadas circunstancias de orden social.

La democracia, como mecanismo de representación, participación y control basado en un conjunto de reglas y normas que le dan sentido, coexiste con una latente insatisfacción respecto de su funcionamiento. No resulta novedoso señalar la fenomenal distancia que pareciera existir entre representantes y representados, entre los temas de interés de la ciudadanía y las preocupaciones de los líderes políticos, la poca capacidad de resolver los problemas ante una marańa de entidades que se trasladan la responsabilidad y la cada vez mayor asimetría de poder y control, todo lo cual nos remite indefectiblemente a la calidad de la democracia como método y práctica; como método de dirimir quién administra el poder y como práctica para determinar la manera en que el poder (recursos) disponible son utilizado en base a objetivos especialmente consensuados. Esta es una línea relativamente nueva de investigación para apreciar el funcionamiento de la democracia y por tanto sujeta a múltiples críticas, partiendo por la posibilidad de medir y qué medir dentro del sistema.

Considerando el exordio contextual previamente señalado, este trabajo se ha planteado como objetivo cardinal, el analizar - a partir de los diversos fundamentos teóricos revisados - los aspectos más importantes relacionados con el terceto conceptual: confianza, capital social y democracia; colocando un énfasis especial en su impacto en el desarrollo. 
Para alcanzar las metas trazadas, se procedió a realizar un análisis documental, lo que llevó a la revisión de diversas fuentes secundarias de información, para obtener en primer lugar las definiciones conceptuales que permitan contar con un marco teórico básico desde el cual iniciar el trabajo reflexivo. Partiendo de dicho punto, se ha contado con estudios de campo para revisar la aplicabilidad de dichos conceptos y su adhesión en términos de población, tanto desde la mirada de las instituciones como de las personas, haciendo los respectivos análisis para obtener elementos que permitan reforzar o refutar las perspectivas iniciales de análisis.

A modo de colofón, se indica que los retos que se derivan del tema desarrollado, son inconmensurables, especialmente considerando la necesidad de que la democracia se consolide a partir de la satisfacción que debe producir en la ciudadanía; hecho que impacta en la estabilidad de los regímenes y la gobernabilidad de los países. Es muy relevante gestar niveles de confianza en la población, todo lo cual debe lograr que las personas se sientan parte plena de la democracia y totalmente incluidos en el progreso que van experimentando los países. En tal sentido, parece axiomático sostener que en paralelo al crecimiento económico, que es sine qua non, también se reflexiones en torno a mejorar la distribución de oportunidades; es decir, se piense prioritariamente en el desarrollo.

\section{Los dominios fundamentales}

Existe una serie de variables que es necesario precisar su alcance, la manera de afectación al objeto de estudio y su conceptualización, para poder luego establecer las relaciones mutuas que se instituyen. En la Figura 1, se puede observar una triada de conceptos, los que han servido de base para realizar el presente trabajo. 
Figura 1 - Triada confianza, capital social y democracia

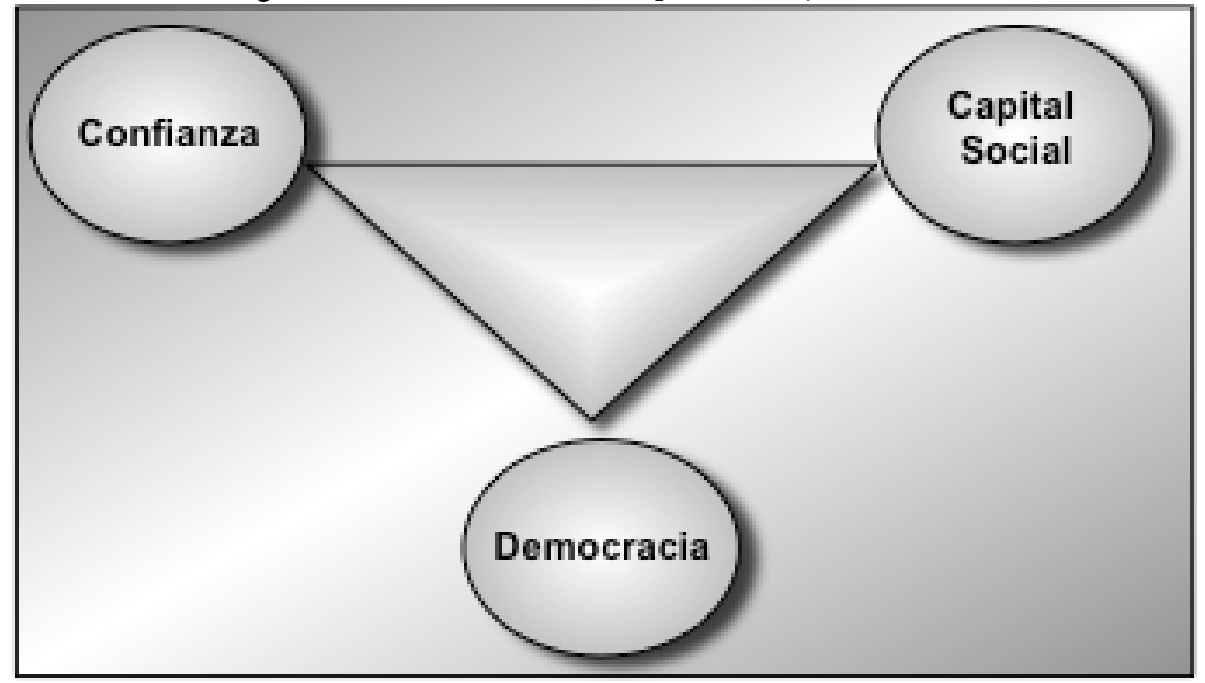

Fuente: Diseño propio, 2015.

Se comenzará el análisis con la confianza, esta conceptualización - para entenderla mejor - se analizará desde la perspectiva social y también institucional, es decir, por una parte se abordará su valoración en la relación existente entre las personas y luego veremos cómo se afecta en tanto entramado normativo, su respeto, reciprocidad y eventual beneficio frente a la posibilidad de caos total o más bien la tendencia al Leviatán en términos políticos.

En cuanto a la democracia el análisis se centrará en aquella idea de satisfacción en tanto evaluación social de ella ligado a la gobernabilidad, a la vez que indagaremos en las nociones de calidad de la misma teniendo algunos indicadores básicos planteados y que nos permiten asociarla a la estabilidad del régimen. Siendo el marco democrático el que permite establecer a una sociedad propósitos comunes, ante los cuales trabajar de manera coligada movilizando recursos de diversa índole, así como valores y principios compartidos que dan sustento a la construcción de capital social, basados en una red de confianza colectiva que se traduzcan en procesos que promueven el desarrollo.

Por otro lado, fomentar el crecimiento económico sin mirar el fortalecimiento de las instituciones también coloca presión a la democracia, especialmente en momentos de crisis, donde el cuestionamiento a su utilidad puede llegar a poner en riesgo su vigencia. El incremento de la economía es un factor esencial, pero a la par con la distribución de los beneficios que promueve la sociedad, si la democracia ofrece beneficios económicos particulares, estos debieran tener una forma de 
medición que esté basado en términos de las oportunidades que se hacen disponibles, más que con la tasa media de crecimiento (KAPSTEIN y CONVERSE, 2008). En este contexto, se debe observar lo que ocurre con aquellos individuos que quedan en el camino de las transformaciones y cómo éstos actúan frente a la democracia, dado que muchas veces ocurre que pierden la confianza en ella, hay desafección, apatía y rechazo, lo que a la larga se transforma en un riesgo para su estabilidad de los países.

\section{Confianza social}

Al iniciar el análisis, se comenzará por abordar el concepto de confianza social, lo que lleva a indagar en un término cuyo significado es algo difuso y por tanto puede significar múltiples cosas a la vez, incluso contradictorios entre ellos, sin embargo, para efectos de entenderlo diremos que la confianza es un "interés encapsulado", como señalara Russell Hardin (MONTERO, ZMERLI y NEWTON, 2008, p. 11-54) ya que ello implica que "sus intereses contienen el mío, eso sería lo que me lleva a confiar en ti”. Aquí se da una noción clásica del enfoque, ya que implica una suerte de promesa cumplida y fundamentalmente de una dependencia mutua, lo que implica disminuir los riesgos al asumir que los otros actuaran de una manera predecible o confiable, podemos decir que hay un acto de fe en los demás al creer en su palabra. Más adelante se analizará el capital social, pero parece oportuno señalar su vinculación con la confianza social, toda vez que ella importa una suerte de letra de cambio o crédito puesto a disposición de otros, los cuales están siempre en situación de ser cobrada. Esto implica un intercambio o la necesaria acción de reciprocidad por parte de quien recibe, es en ese sentido que se entiende el concepto de crédito. Dónde entra la confianza se preguntará, en el ambiente social, donde se producen por una parte; y en las posibles garantías de saber que ello será cubierto por parte de los actores involucrados como segundo elemento adjunto.

La confianza que se da entre dos agentes asume que ninguno de ellos se dañará ni actuará en su contra de manera consciente en su acepción mínima; y que en el otro extremo supone que uno de los agentes intentará proteger al otro o aumentará sus logros basado en los intereses que reconoce en su contraparte. Esta es una suerte de ganar-ganar donde se actúa de manera de potenciar favorablemente los intereses comunes, reconociendo que parte de los intereses están bajo la responsabilidad de otro, ante lo cual se está dispuesto a asumir el riesgo del cumplimiento de la promesa. Por lo tanto, podría afirmarse que la ausencia de confianza es nítidamente incompatible con el adecuado funcionamiento que se demanda de una sociedad (JUDT, 2010). La reflexión de Judt, ahondaba en los resultados de dicha confianza en términos de cohesión y homogeneidad de la misma sociedad. Pareciera entenderse 
en dicho concepto la noción de una sociedad donde existe un conocimiento de sus miembros, aquellos códigos compartidos mediante los cuales se reconoce y se es parte de un todo que es la sociedad de la cual se forma parte.

Como es sabido, varias investigaciones han centrado su análisis en el fenómeno de la confianza y a manera de muestra, se puede apreciar en el Figura 2, que cuando se le pregunta a las personas en Latinoamérica si es posible confiar en los demás, la respuesta es en general negativa (teniendo un promedio de $22 \%$ ); con casos extremos como los de Brasil, Nicaragua y Chile que llaman la atención por lo bajo de su aceptación, situándose en 9\%, 15\% y 17\% respectivamente. Este indicador, tomado de Latinobarómetro nos permite ver una secuencia de diversos ańos y su variación en el tiempo, sorprende no solo lo bajo de la cifra, ya que en años como el 2000 y 2004 llegó a $16 \%$ en promedio regional. Al exponer dichos datos en comparación con otras zonas del planeta, el mismo informe en su versión 2011 resalta que "este mismo indicador alcanza niveles cercanos a los 70\% en los países europeos, manteniéndose ésta como una de las principales diferencias entre nuestras sociedades" (LATINOBAROMETRO, 2012). Advertimos desde ya un factor a considerar al momento de evaluar el funcionamiento de la democracia y las posibilidades de alcanzar un desarrollo adecuado y fundamentalmente equilibrado entre los distintos miembros de la sociedad. Esta realidad está muy lejos de acercarse a la propuesta de sociedad civilizada que hace el filósofo Avishai Margalit, cuando sostiene que hay que avanzar no sólo hacia la sociedad justa, sino, principalmente hacia una sociedad decente, y la entiende como aquella que no humilla a sus componentes (BUROTTO y GANGA, 2012). 
Figura 2 - Confianza interpersonal como \% de aprobación

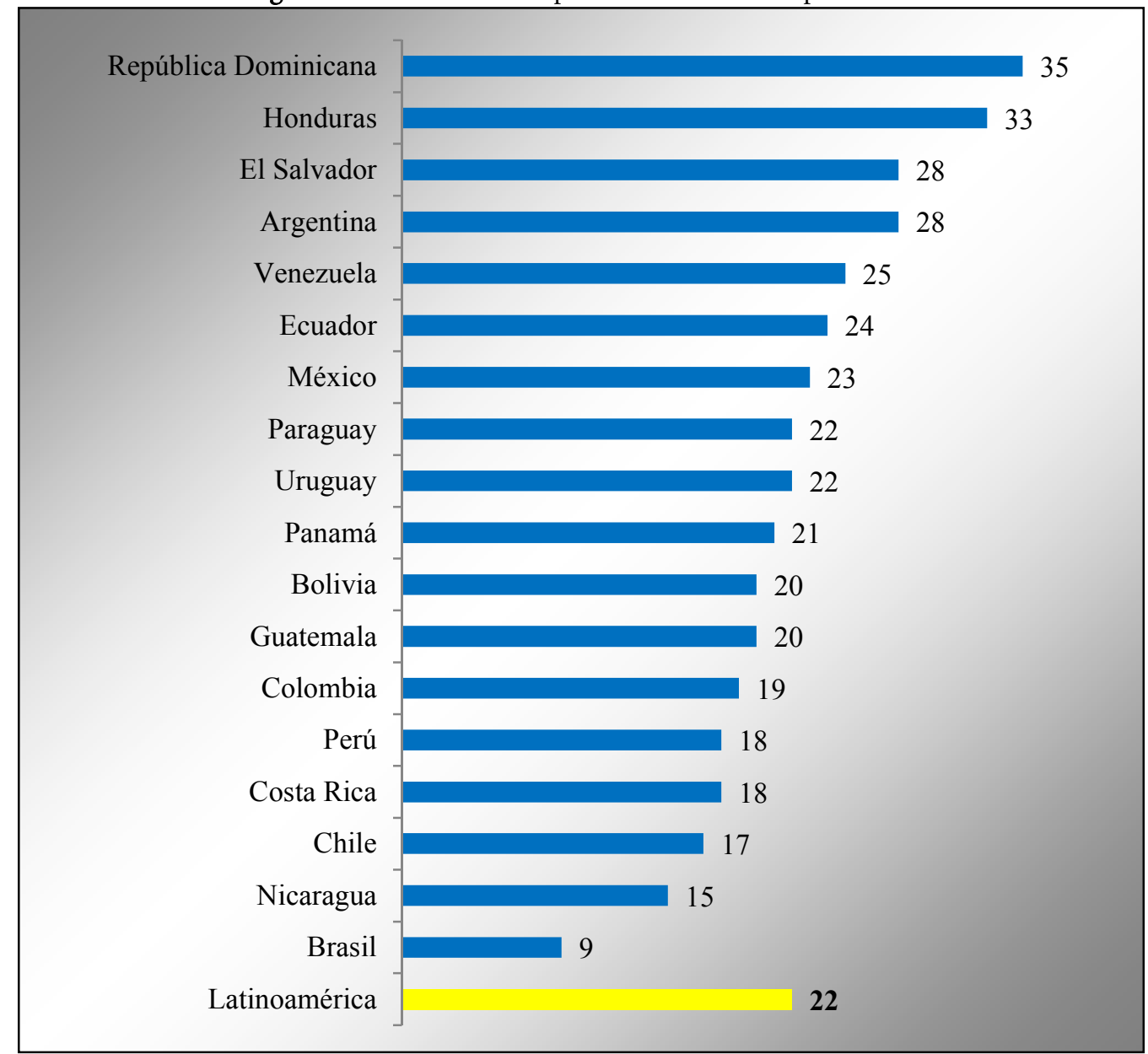

Fuente: Diseńo propio, 2015, basado en Latinobarómetro (2012).

\section{Confianza en las Instituciones}

En el caso de la confianza en las instituciones, podemos iniciar el concepto con una prolongación de la confianza social, lo que nos lleva a asumir que ellas actuaran de una forma predecible, no arbitraria ni menos dañina para los intereses de la sociedad o el país, tratando a los ciudadanos de manera igualitaria, correcta y justa. Siguiendo a Guillermo O'Donnell (1996, p. 5-28) al respecto nos advierte que la institución implica "un patrón regularizado de interacción que es conocido, practicado y aceptado (si bien no necesariamente aprobado) por los actores que tienen la expectativa de seguir interactuando bajo las reglas sancionadas por ese patrón”. Por cierto que ello implica la valoración que las personas hacen de las instituciones centrales del sistema político, y aquí existe uno de los primeros 
elementos a indagar en el actual contexto latinoamericano, ya que ello supone distinguir algunos atributos que extrapolamos de los individuos como la credibilidad, transparencia, imparcialidad, equidad y honestidad en sus planteamientos u objetivos explicitados, recordemos que hay una suerte de sino compartido entre la persona y la institución, al romperse ese destino común la confianza se desmorona.

Hace ya varios años el mismo estudio del Latinobarómetro han medido la confianza en las instituciones, lo que nos permite revisar la evolución del indicador en un período extenso de tiempo, mostrándonos un alto grado de desconfianza existente en los países de la región. Para efectos de este estudio hemos seleccionado sólo aquellas instituciones de carácter indiscutiblemente políticas, las que se muestran en el Figura 3, lo que nos puede ayudar a entender la vinculación con la satisfacción con la democracia, ya que podemos ver que ninguna de ellas supera el 50\% y en el límite inferior se encuentran los partidos políticos que rondan el $20 \%$.

Destaca en los datos aquellas instancias que por su mandato o por su estructura tienen una mayor capacidad de hacer, como son los Gobiernos y los Municipios/Gobiernos Locales, ello pareciera ser mejor percibido por la población ya que se ve una respuesta concretar a sus demandas, lo cual podría estar más cerca de satisfacer o más bien se asemeja a la idea que la gente tiene de lo que deberían realizar estas instituciones, es decir de generar gobernabilidad a distintos niveles dentro de los países. 
Figura 3 - Resumen Confianza en las instituciones Promedio América Latina 2001-2011

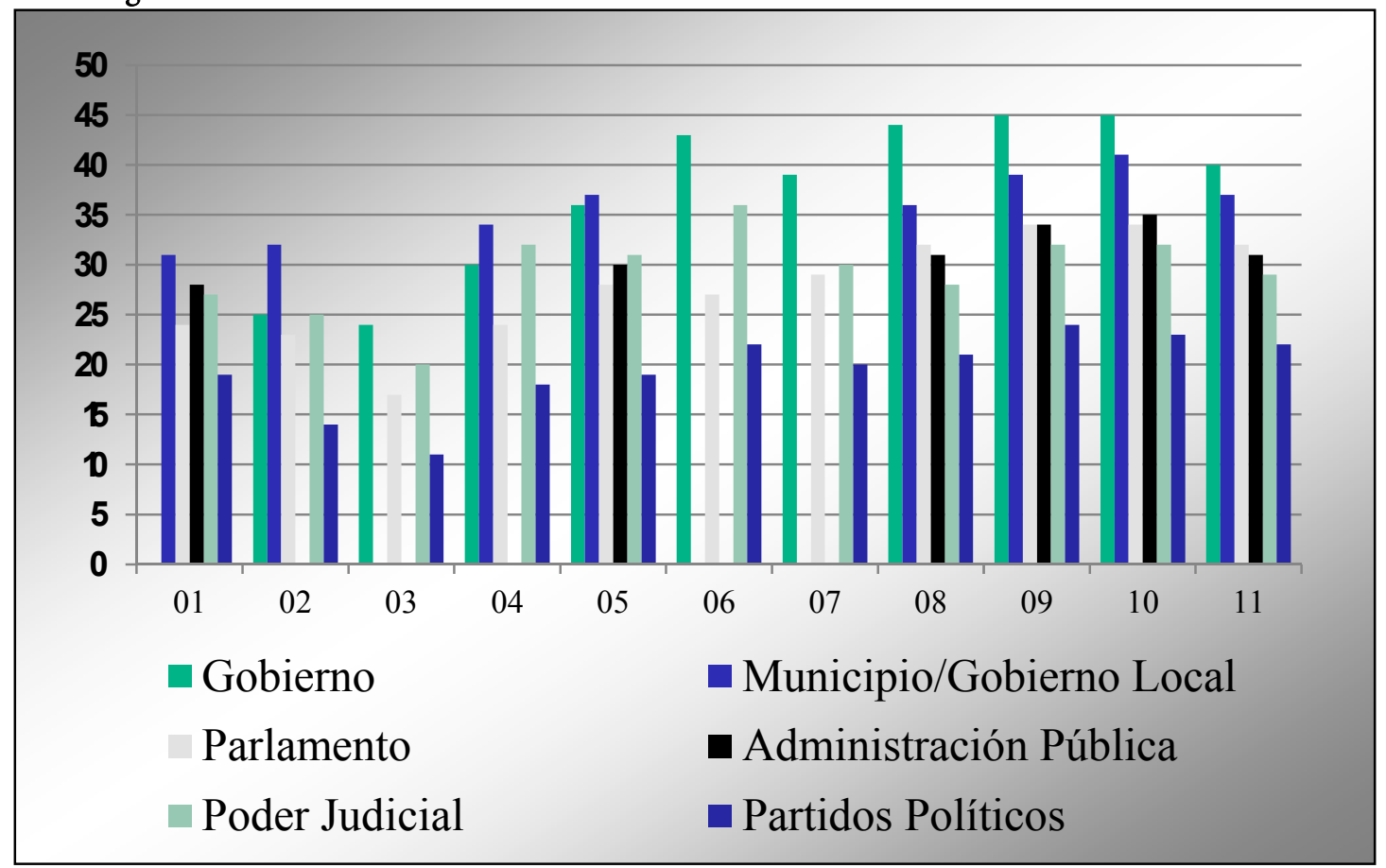

Fuente: Diseńo propio, 2015, basado en Latinobarómetro (2012).

Estos datos no parecen sorprender al ver también los porcentajes de confianza interpersonal entre ciudadanos mostrados en la Figura 1, los cuales parecen reproducirse cuando vemos la confianza en las instituciones, especialmente en aquellas de libre asociación como son los Partidos Políticos.

\section{Satisfacción con la democracia y su calidad}

La satisfacción con la democracia está asociado a la posibilidad que tiene ésta de dar respuesta a las demandas de la gente, ello implica una evaluación por parte de la población de la actuación de los líderes políticos que están al mando de las instituciones públicas. En este punto se puede hacer una distinción entre la satisfacción respecto del régimen democrático frente a la respuesta asociada a las políticas públicas que implementa un gobierno. En el primero nos haremos cargo de la estabilidad del sistema, lo cual es menos variable en el tiempo, sin embargo, el segundo aspecto implica el concepto de gobernabilidad, el que está mucho más vinculado con la satisfacción que percibe la gente y este concepto es mucho más volátil. La legitimidad del sistema político se percibe frente a la satisfacción como 
capacidad de responder mediante el diseño acertado y la implementación satisfactoria de políticas públicas.

Reconociendo que no todo los sistemas sociales gobernables son democracia, si podemos asumir que:

[...] es gobernable, entonces, cuando está estructurado sociopolíticamente de modo tal que todos los actores estratégicos se interrelacionan para tomar decisiones colectivas y resolver sus conflictos conforme a un sistema de reglas y de procedimientos formales o informales - que pueden registrar diversos niveles de institucionalización - dentro del cual formulan sus expectativas y estrategias (CATALÀ, 2001, p. 120).

Estas relaciones y sus decisiones las podemos definir como democráticas cuando se producen basadas en dicho concepto, con el cual nos alejamos del Estado Leviatán al cual alude Hobbes y que fue publicado originalmente en 1651.

Podemos asumir la existencia de una relación directa entre gobernabilidad y democracia, ya que esta conexión supone el traslado del mandato electoral a políticas públicas concretas, es decir supone una relación entre las soluciones - respuestas - de los representantes a las demandas planteadas por los representados - ciudadanos -, lo cual es esencial en una democracia, pero prescindible en un sistema no democrático.

Para el caso chileno se ha decidido tomar los antecedentes mostrados por el estudio de opinión realizado por el Centro de Estudios Públicos (CENTRO DE ESTUDIOS PÚBLICOS, 2012), y tal como puede observarse en la Figura 4, los datos no difieren de lo entregado en promedio por el informe de Latinobarómetro, en este orden de cosas, se podría afirmar que refuerzan la tendencia y marcan con más nitidez la caída en las instituciones eminentemente políticas. Especial atención se debe poner a los partidos políticos y el congreso, ya que por su carácter, su impacto en la valoración que se hace de la democracia es relevante. Estos datos son sobresaliente - ya que puestos en perspectiva - permiten vincularlos con el siguiente concepto, cual es la satisfacción con la democracia, y en este orden de cosas, es muy interesante apreciar el efecto que se tiene al ver los niveles de apoyo con que cuentan. 
Figura $4-$ ¿Cuánta confianza tiene Ud. En cada una de ellas?

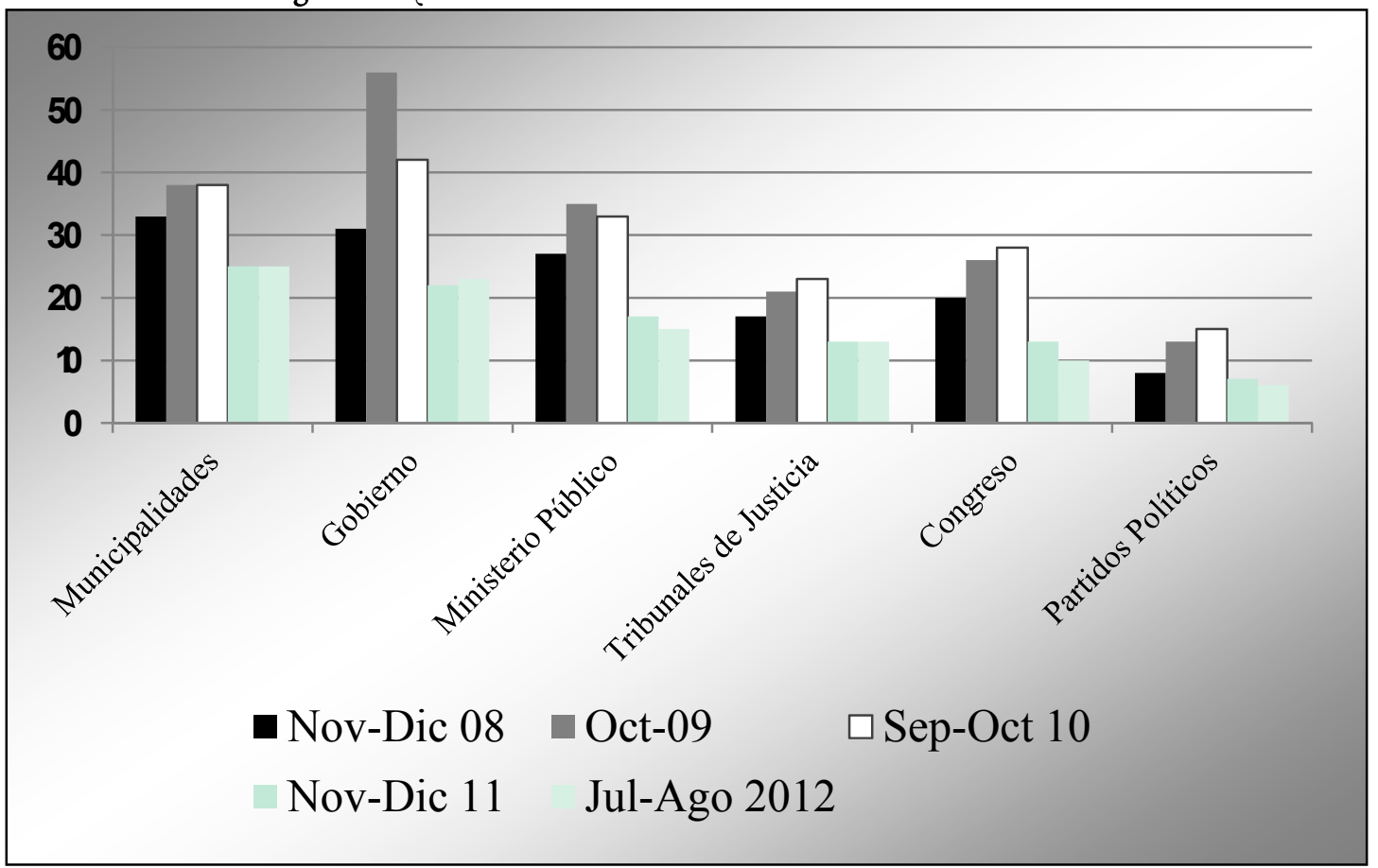

Fuente: Centro de Estudios Públicos, 2012.

Considerando lo expuesto previamente, es factible afirmar que en las complejas sociedades actuales, donde se inserta la democracia contemporánea "puede afirmarse que existe una relación entre las instituciones democráticas de una sociedad y la eficacia política de sus gobiernos: así como entre ese diseño institucional y la consolidación, estabilidad o gobernabilidad de las democracias" (VILLARROEL, 2000, p. 9-35). Es materia de análisis verificar si existe y cómo se da la relación entre la vigencia de una democracia de calidad, la gobernabilidad, la presencia de instituciones que son capaces de cumplir con sus cometidos, dando respuestas a las demandas generadas por la población y asumidas por el Estado.

Se puede entender la democracia desde una perspectiva jurídico-formal, en la que funciona como un "sistema político que convierte la expresión de la voluntad popular en normas vinculantes para todos los sujetos políticos y para todos los poderes estatales" (VELASCO, 2006, p. 40). Desde dicha visión, la participación y fundamentalmente la comprensión de la discusión política, por parte de la ciudadanía se hacen evidentes. Esto tiene que ver con la capacidad inclusiva de la institucionalización política, la cual debe ser capaz de generar los espacios para el 
debate deliberativo de ideas entre las distintas organizaciones y ciudadanos que componen la sociedad.

Esta democracia importa que sea respaldada por los ciudadanos, estos perciban algún grado de satisfacción respecto de su existencia y la ciudadanía asuma un compromiso cívico con ella. Lo que eventualmente se podría traducir en participación política de manera directa $\mathrm{o}$ al menos en el reconocimiento de instituciones que le dan sustento a la democracia como son los partidos políticos, el congreso o el gobierno. La democracia de calidad podría ser entendida como "aquel orden institucional estable que permite la realización de la libertad e igualdad de los ciudadanos a través de instituciones o mecanismos adecuadamente funcionales" (MORLINO, 2008, p. 1-24).

Siguiendo con el análisis que Morlino realiza sobre la calidad de la democracia la cual separa en tres instancias, señalando en primer lugar que la calidad está referida a sus resultados, una segunda calidad está asociada a su contenido y la tercera respecto de los procedimientos.

Tal como puede visualizarse en la Figura 5, Morlino sostiene que una buena democracia "tiene por lo menos seis dimensiones de variación que tienen que colocarse en el centro del análisis empírico y que pueden ser consideradas como sus calidades" (MORLINO, 2007, p. 3-22). Las primeras tres son de carácter procedimental y en ellas tenemos el respeto a la ley (rule of law), responsabilidad electoral (accountability electoral) y responsabilidad inter-institucional (accountability inter-institucional); en tanto la reciprocidad (responsiveness) es la dimensión que está asociada con los resultados "en el sentido de la capacidad de respuesta que encuentra la satisfacción de los ciudadanos y de la sociedad civil"; por último la libertad y la igualdad que son de carácter sustantivo. 
Figura 5 - Dimensiones de la democracia

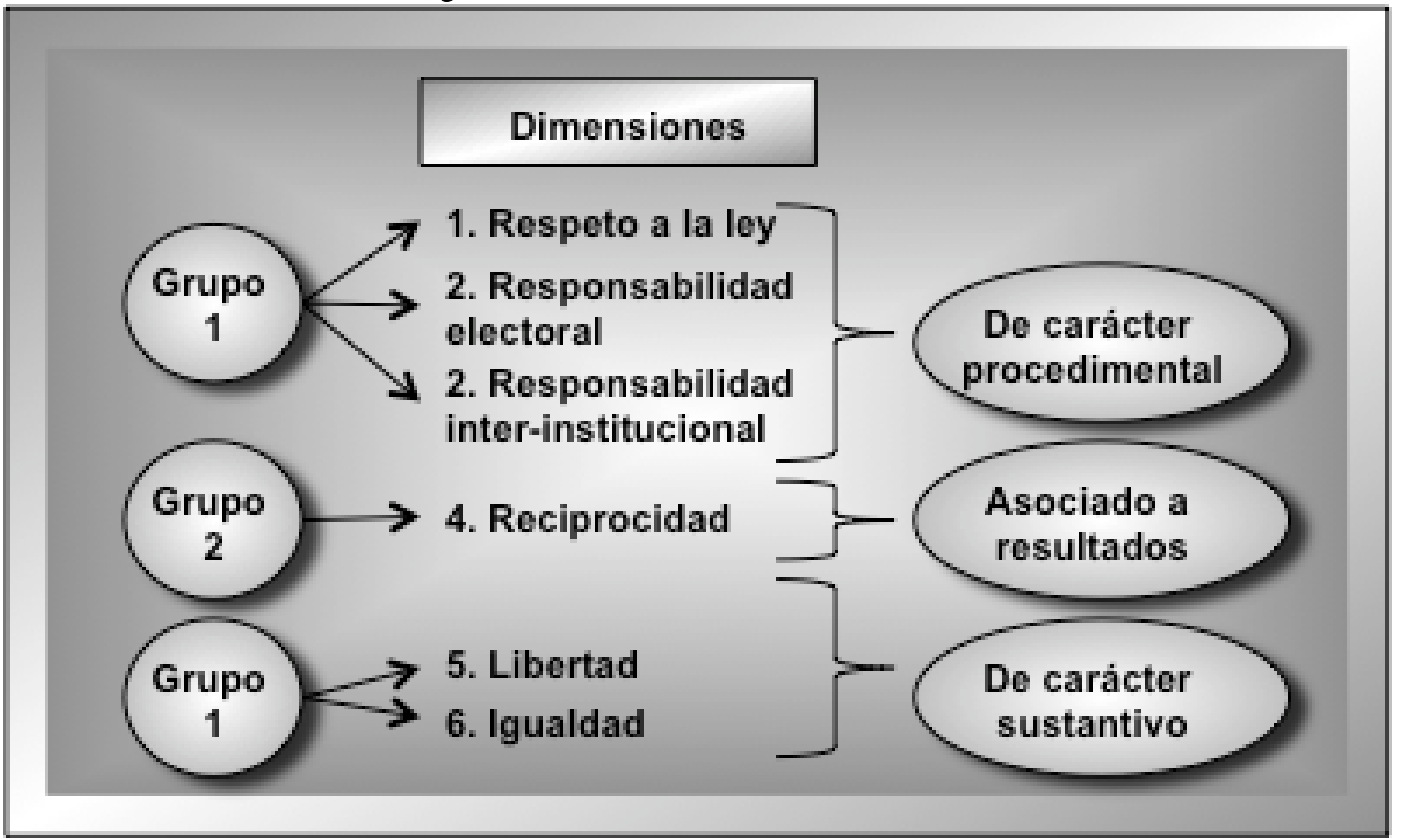

Fuente: Diseńo propio, 2015, basado en Morlino (2007).

Un factor que debemos incorporar en nuestro estudio para entender esta vinculación entre calidad y democracia o mejor dicho esta adjetivación de la democracia es la gobernabilidad, entendiendo que las acciones del gobierno, su grado de eficiencia, efectividad y oportunidad no son indiferentes para las personas y en ese sentido no da lo mismo quien gobierna. Uno puede entender en dicha frase un mensaje referido a que no da lo mismo como el gobierno de turno gestiona los recursos del Estado, dónde están puestos los énfasis de las políticas públicas, cuál es el trasfondo ideológico o desde dónde se mira a la sociedad y se construyen los acuerdos societales.

En dicho sentido la gobernabilidad aparece vinculada a la democracia, ya que esta se reconoce cuando un gobierno está en condiciones de tomar decisiones, llevarlas a cabo de manera coherente y coordinada con los objetivos propuestos en su momento y son capaces de cumplir con las demandas que la ciudadanía tenía cuando estas se gestaron. En la medida que este proceso se desarrolla y consolida se transforma en un factor de legitimación del sistema político donde las personas perciben que sus intereses son agregados y solucionados mediante acciones efectuadas por órganos del Estado o normados, de tal manera que su accionar está debidamente protegido, hay una certeza mediante la cual se desempeñan sus actividades. 


\section{Capital social}

Entendidos los conceptos anteriores, es factible derivar hacia el capital social, el cual implica combinar la confianza social y la confianza en las instituciones. Parafraseando a Jordana (2000), al considerar la variedad de entidades, es preciso reconocer dos características relevantes:

- Todas consisten en un cierto aspecto de la estructura social y;

- Todas facilitan ciertas acciones de los individuos que están dentro de la estructura.

El punto de intersección de las variables mencionadas y el capital social es la confianza; dándose un continuo entre la confianza social y la institucional que permite construir capital social, lo que sería el gran resultado de su existencia y por ello la importancia de fomentar todo aquel mecanismo que aumente su presencia.

En el caso de Putnam (1995), parece quedar más claro al considerar la confianza interpersonal como un factor que facilita la cooperación entre los individuos, reduciendo los requisitos para dicha cooperación, lo que nos recuerda los costos de transacción que se generan en toda sociedad, tal como North lo expresara en sus estudios de economía. Dicho costo de transacción lo toma Fukuyama (1996), al comparar a aquellas sociedades donde la confianza está presenta frente a aquellas donde es un valor escaso, lo cual representa una especie de impuesto que perjudica a todos, es en este caso donde las instituciones cobran un mayor valor o se hacen imprescindibles no por ser una creación espontánea de la sociedad, sino más bien por lo contrario, ya que la gente que no confía en su prójimo termina cooperando con éste bajo un sistema normativo y coercitivo. Lo que nos lleva a considerar nuevamente al Estado como aquel garante de los acuerdos y normas bajo un sistema democrático, el que tendría la función de suplir la ausencia de confianza.

Se puede agregar que parte de esa reducción de requisitos puede estar dada por instituciones que ayudan a resguardar las acciones de las personas sin caer en contradicción con la confianza, ya que la refuerzan y generan un círculo virtuoso dentro de la comunidad. Reconociendo que esas instituciones pueden ser de carácter formal o informal, lo relevante es la capacidad de identificar normas sociales o pautas de comportamientos que son reconocidas y aceptadas por los miembros de una comunidad, ya que su respeto da continuidad a las relaciones, a la vez que generan sanciones en caso de transgredirlas aislando a aquellos que caen en tal situación o estableciendo mecanismos de coerción también reconocidos por todos para dirimir los conflictos de intereses incrementando los costos potenciales para aquellos desertores del sistema en el cual participan. 
Hay que entender que las relaciones de causa entre sociedad y política van desde la primera a la segunda y no al contrario, implicando el necesario entendimiento de la manera en que esa relación se produce, como se concatena esa trasmisión de intereses desde la sociedad a la política para que esta última los modifique, reforzando la necesidad de comprender a las instituciones políticas, ya que serían esas las catalizadoras de las demandas de la sociedad y en un plano de confianza, los individuos evalúan la respuesta que surgen de su accionar en un contexto de eficiencia y oportunidad.

\section{Integración de conceptos}

No es de extrañar por tanto, que en un estudio publicado en la Reis del 2008, el cual mide la asociación entre confianza social y confianza en las instituciones políticas exista una correlación significativa y "su potencial importancia teórica para la teoría del capital social” (MONTERO, ZMERLI y NEWTON, 2008, p. 25). Este estudio cubre una muestra de 13 países europeos y sus resultados confirman que la "confianza social se encuentra asociada fuerte y consistentemente con la confianza en las instituciones políticas en el nivel individual" (MONTERO, ZMERLI y NEWTON, 2008, p. 32).

Esto es interesante a la luz de la teoría de Bourdieu, donde el capital social sería aquello que diferencia a personas que tienen el mimo nivel económico, el mismo nivel cultural, pero que obtenían diferentes beneficios, lo que estaría dado por las relaciones o la capacidad que tienen unos individuos de "movilizar a su favor el capital de un grupo más o menos institucionalizado y rico en capital" (PLASCIENCIA, 2005, p. 23), lo que no es más que su red de vínculos que le permiten reconocimiento, aceptación para realizar su labor y pertenencia a un grupo determinado. Pasar del individuo a la institución en este caso importa, ya que nos debemos fijar en el capital que posee la institución (grupo) de la cual forma parte, debido a que es ese el capital que movilizará o le permitirá instalarse, es una suerte de apropiación de dicho capital institucional por parte del individuo para beneficio propio pero también para retroalimentar a la organización de la cual forma parte, por eso es una relación simbiótica de crecimiento mutuo, de acumulación de poder para mostrar, pero también para desarrollar nuevas acciones.

Es reiterado apreciar que el capital social se vincula con la existencia de normas, valores y redes que dan cuenta de un conjunto de recursos que están a disposición o como mejor señala Putnam (1995), mecanismos de la organización social como redes, normas y la confianza social, facilitan la coordinación y cooperación para beneficio mutuo y reconocen los elementos esenciales del capital 
social, pero también permiten visualizar que estas relaciones atañen una reciprocidad y una implicación de las acciones que las personas o instituciones emprenden.

Aquí se puede volver la mirada a la calidad de la democracia como la postula Morlino (2008) con ideas fuerzas que tienen el accountability y el respeto de la ley como elementos básicos; pero también están los estudios de Levine y Molina (2007) en el que exponen una situación en base a variables verificables. Ellos han propuesto cinco dimensiones para su definición de calidad de la democracia los cuales se centran en elementos conceptuales, los que se muestran graficamente en la Figura 6.

Figura 6 - Dimensiones para definir la calidad de la democracia

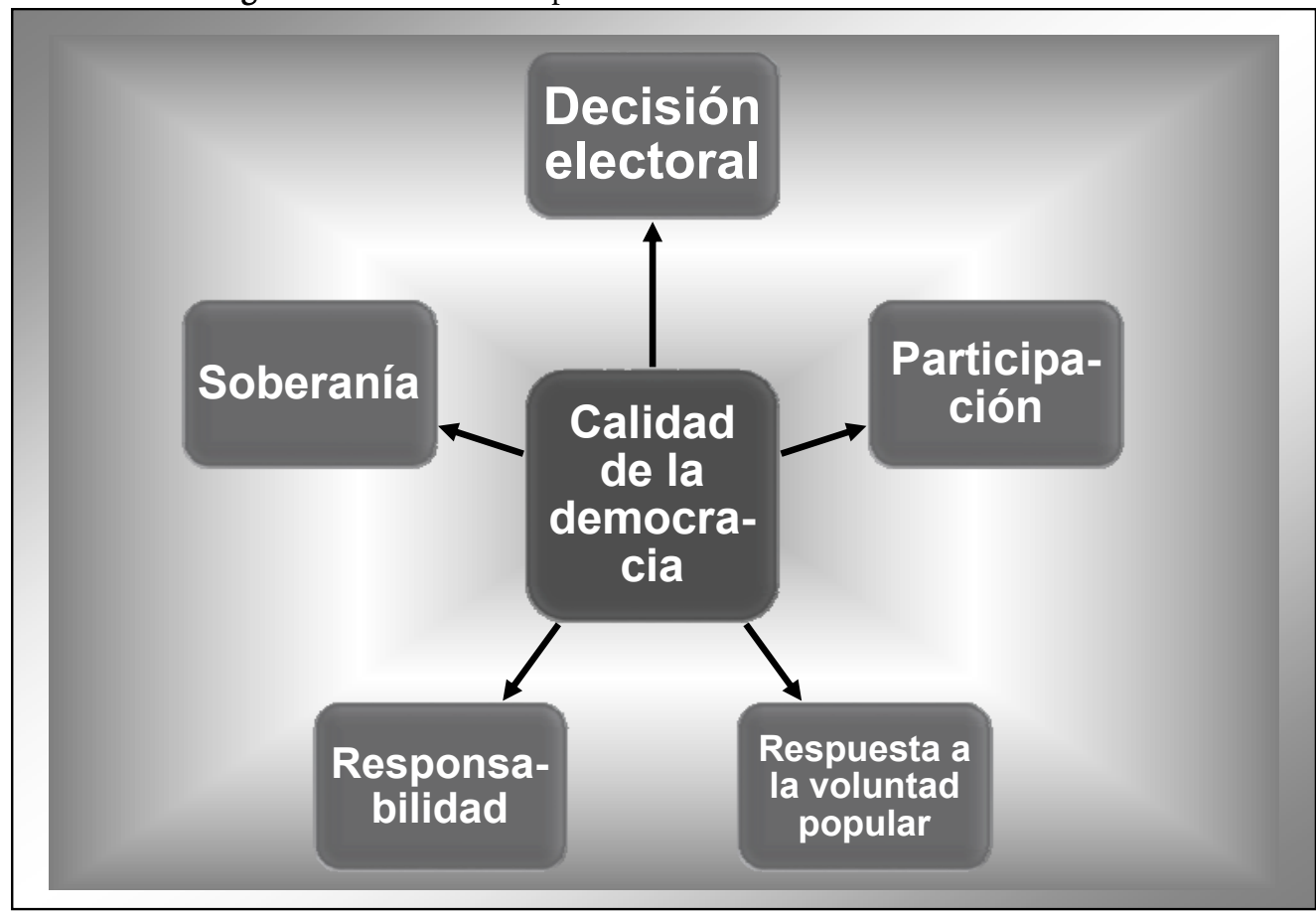

Fuente: Diseńo propio, 2015, basado en Levine y Molina (2007).

Por otra parte, se debe reconocer que ciertos grupos sociales pueden considerar de mejor manera su relación con la comunidad, el asunto es que se debe especificar a qué comunidad se refieren y ese sería uno de los problemas en sociedades menos integradas o donde los beneficios creados no son distribuidos de manera equitativa, lo que es un problema ya que existe un factor de apropiación de los beneficios por parte de quienes los producen, lo cual es legítimo y nuevamente nos enfrentamos a la necesidad de contar con instituciones políticas que contribuyan a la creación de mecanismos de inclusión, sea por la vía educacional como por la vía de 
interacción de individuos de origen diferente en instituciones que cuenten con capital para sus miembros, por ejemplo los partidos políticos, iglesias, organizaciones gremiales o los sindicatos como forma tradicional hoy hablaríamos de organizaciones no gubernamentales de todo tipo, que agrupan a las personas más bien por intereses compartidos y de carácter voluntario. Sin embargo dicho espacio es muy reducido para distribuir el capital social, especialmente en aquellas sociedades donde la idea de asociatividad es baja.

Sería razonable esperar que el capital social esté relacionado con la confianza social y política en el plano individual y en el agregado. Lo que no es tan claro es que la confianza individual, esa confianza que se deposita en el otro, en un igual, tenga relación directa con la satisfacción en la democracia; por tanto, se puede inferir que serán las instituciones las que agregan valor a una sociedad y es en ellas en quienes los individuos radican las responsabilidad para acrecentar su satisfacción frente al orden político establecido mediante un régimen democrático.

Esta no sería una cuestión menor al considerar que el sistema político en general y la democracia en particular, permite distribuir al menos una parte del poder que tiene una sociedad a través de sus diversas instituciones aumentando los niveles de pertenencia e inclusión social que proporciona la interacción entre los distintos agentes involucrados. Por ello, la democracia es vista como "un sistema de representación, con participación libre y universal de la población adulta en un marco de igualdad de derechos y reglas del juego también iguales" (LEVINE y MOLINA, 2007, p. 19).

A esta altura surge la interrogante de por qué seguir hablando de capital social, ya que en muchos casos lo que tenemos es una serie de relaciones que se fundan en la confianza, sea individual sea institucional, bajo un marco normativo que es la democracia y que pretende resolver problemas que le son común a un grupo de personas, frente al cual se agrupan. Pues bien, es social porque "proviene de la interacción entre agentes $\mathrm{y}$, segundo, porque tales interacciones o relaciones, ingrediente básico de todo capital cualquiera sea su contenido, genera nuevas realidades sujetas a apropiación social; esto es, genera diferencias relacionales entre individuo y grupo" (LOZARES et al., 2011, p. 6)

El grado de colaboración puede ser un indicador de la profundidad o penetración de la confianza en una sociedad, en términos prácticos tal colaboración permite disminuir los niveles de incertidumbre que se da en la sociedad y con ello las diferencias existentes, como ya señalamos en materia económica esto se traduce en los costos de transacción, pero también lo podemos visualizar en las actividades de 
innovación colaborativa o las mismas redes de investigación que se generan en la universidad.

Como una forma de esquematizar la interacción de los diversos componentes o elementos analizados, se diseñó la Figura 7.

Figura 7 - Integración de componentes

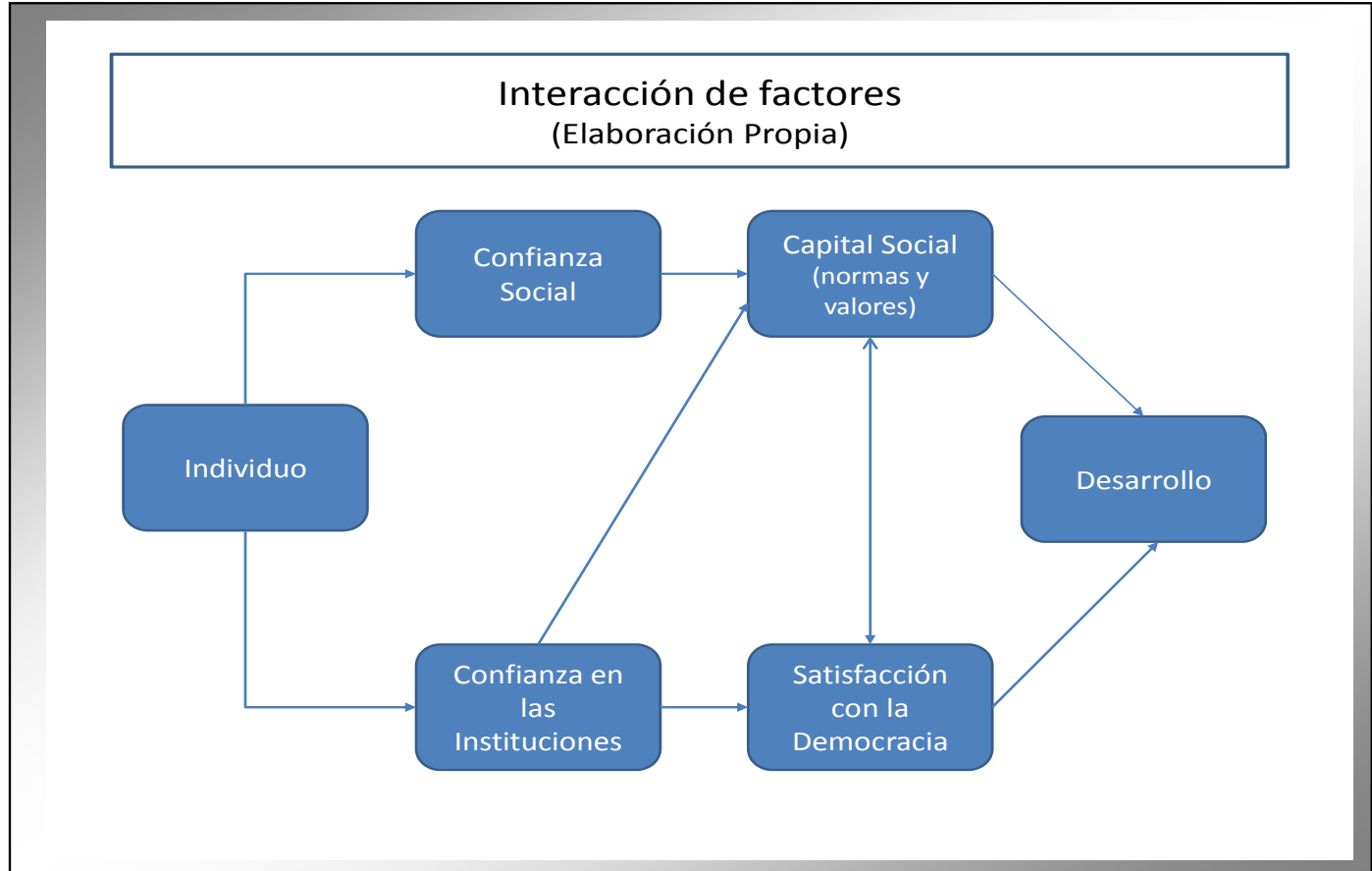

Fuente: Diseńo propio, 2015.

En tanto organización, al acceder a sociedades con altos niveles de confianza es factible estructurar las organizaciones de manera flexible y con una clara orientación al resultado, donde el trabajo en equipos colaborativos es recurrente y los niveles de delegación son altos. Por el contrario, en aquellas sociedades donde no se posee la responsabilidad suficiente para acatar normas se hace necesario crear un sistema de regulación que desvía recursos, limita la relación entre los miembros.

\section{Democracia impactada}

Luego de analizar detenidamente las diferentes variables es posible asumir que el capital social resulta intransferible, por lo tanto este se vincularía de manera directa a una sociedad (país), lo que nos lleva a pensar que su impacto en la condición que asume la democracia también será particular y representativo de una sociedad establecida. 
La democracia implica entre muchas otras cosas, una manera de distribuir el poder, como el capital social es en parte poder de un grupo, su relación se hace importante en la medida que implica la apropiación por parte de unos pocos pero ratificados por la mayoría de la administración temporal de la misma sociedad, como diría Jensen y Meckling (1976), se da nítidamente una relación contractual entre agente y principal, los cuales tienen ópticas disímiles de los fenómenos organizacionales (GANGA y BUROTTO, 2012); por cierto que su relación con la gobernabilidad es directa, ya que ello lleva al fortalecimiento del sistema de respuesta coordinada y efectiva que se implementa. Esto es lo que permite traducir crecimiento económico en beneficios sociales, por ejemplo, lo que lleva a equiparar en parte el capital social de los grupos que han quedado fuera.

En la medida que las personas piensan que la democracia y sus reglas les permiten acceder o al menos moverse por la estructura social, alcanzando beneficios, se valida como forma de organización social y potencia una vía inclusiva de desarrollo, aumentando el compromiso cívico con el régimen. Pasamos así desde la participación en pequeñas organizaciones donde nos conocemos (confianza social) a respetar instituciones a las cuales adherimos por cuanto las consideramos necesarias como es un sistema político en particular o la democracia para ser más específicos de una institución formal que tiene normas y regulaciones bien definidas con pautas de comportamiento y mecanismos de participación que son asumidos por todos los miembros.

Ahora bien, la intervención del Estado no es neutra en ningún caso y puede contribuir o desalentar la capacidad de construir capital en la medida que existe una dependencia mutua entre los individuos, como ya señalamos, esta interdependencia es básica cuando tratamos de mostrar la existencia de relaciones de colaboración, pero si el Estado se involucra mediante acciones de asistencialismo, se pierde dicho vínculo entre los individuos. Es por ello que esta meta organización debe evaluar su accionar para estimular por una parte las valoraciones de las redes versus la pasividad con que algunos esperan la ayuda que viene por los canales institucionales del Estado, destruyendo de paso las potenciales oportunidades de movilidad que se generan en la sociedad.

Lo que pareciera no estar en discusión, por lo menos hasta el momento, es que los Estados eficaces son relevantes y determinantes para una gobernabilidad democrática exitosa y también, para el logro del progreso y desarrollo de los pueblos (MAINWARING y SCULLY, 2008). La afirmación anterior, vuelca nuevamente la mirada a la capacidad de gobernar por parte de los líderes, respondiendo a las expectativas que la población tiene de su función. 
Complementariamente, se debe reconocer que no es posible aislar los derechos políticos de los derechos civiles sin afectar el funcionamiento de la democracia, si situamos dichos derechos en el plano del capital social se puede percibir la relevancia que tienen al incentivar el ejercicio del gobierno de manera eficiente. Al vincularlo con el desarrollo, no faltan los estudios que promueven la supresión de ciertos derechos con miras a privilegiar el desarrollo económico, especialmente en las denominadas etapas tempranas, sin embargo ello lleva aparejado eliminar de la cultura cívica factores fundamentales que luego se transforman en obstáculos para el mismo desarrollo.

\section{Conclusiones}

Independiente de la posición política desde donde se quiera mirar, las instituciones se han convertido en factores fundamentales para transferir las demandas que las personas expresan en la sociedad, puede haber variaciones en los mecanismos, y en ese caso surgen las críticas a la democracia como método, pero no parece existir una variante muy grande respecto de la utilidad.

En la medida que las sociedades van satisfaciendo las necesidades primarias de su población, se hace más patente avanzar en elementos de orden cualitativo como la cohesión social, lo que se ve reflejado en la capacidad que tiene la sociedad de generar movilidad en su interior, así como en la permeabilidad que tienen las élites de recibir y aceptar personas que no son originarias de los grupos dominantes. Esto se encuentra enraizado en la confianza, puede ser ese uno de los factores que impiden a los países latinoamericanos desarrollarse de manera inclusiva.

Se sigue atado a una lógica de clases que es difícil de romper, aun cuando los ingresos económicos se acrecienten. La confianza permite que las organizaciones sean más flexibles y se accione mucho más la colaboración en su interior. La democracia no escapa a esta idea, las disputas por el poder se establecen en períodos determinados, fundamentalmente electorales, luego la intensidad decae de manera significativa para dar paso a la colaboración, en la región esta idea parece no cristalizar.

También se debe reconocer que en la medida que las instituciones no son percibidas como efectivas en la transmisión de las demandas a los responsables, pensemos en la función de los partidos políticos, la ciudadanía se vuelca de manera inorgánica a las calles para manifestar su opinión, es la sensación de un desborde institucional, lo cual debe hacer pensar a los líderes de las organizaciones para indagar en las causas de esta situación que hace que las demandas no se incorporen en la 
agenda de manera ordenadas o planificadas, sino más bien como reacción a la presión que se manifiesta mediante la protesta.

Esto vendría a ser una muestra clara de la desconfianza de las personas, fundamentalmente en las instituciones que deben representarlas y el surgimiento espurio de organizaciones que asoman de manera espontánea para cumplir con dicha labor, pero que no tienen la mirada inclusiva necesaria para lograr instalar una demanda desde la perspectiva del desarrollo social, ya que sólo contempla el logro de objetivos puntuales y acotados a segmentos muy específicos de la población. Esta segmentación de demandas puede llevar a la sociedad a generar respuestas dependiendo de la presión que ejercen los grupos, dejando a los "sin voz" acorralados en un rincón olvidado lo que va en contra del ideal de cohesión social.

Sin duda la expresión de la sociedad es una muestra de participación especialmente cuando se manifiesta por problemáticas de orden público, sin embargo entraña un peligro no menor, que es la individualización de la demanda, lo que va en contraposición total con lo que venimos señalando de la confianza sea en el otro o en las instituciones.

Como respuesta, quienes están en capacidad de tomar decisiones y orientar a la sociedad debieran abrir los espacios y generar los mecanismos de participación, muchas veces modificando sus estructuras e incorporando nuevas maneras para aprovechar todo el caudal de ideas provenientes de la propia sociedad para satisfacer sus demandas. Esta es una interpelación a la misma democracia, la cual debe mejorar sus vínculos con la ciudadanía y motivar su participación, no dejar que corra por carriles separados que pueden segmentar la sociedad en grupos contradictorios entre sí que limitan la posibilidad de acuerdos.

Por tanto, es necesario seguir indagando en la conexión existente entre confianza en las instituciones y la capacidad que tienen éstas de canalizar soluciones en la medida que se les demande. Lo importante es lograr un entramado institucional que permita canalizar y resolver las demandas que se le planteen por parte de la población y que estas retribuyan mediante niveles adecuados de confianza, podemos decir que este sería un círculo virtuoso en la medida que exista.

Por tanto los desafíos son múltiples en consideración a la democracia y la satisfacción que ella debe generar en los ciudadanos, pensando fundamentalmente en la estabilidad del régimen y la gobernabilidad mediante el desarrollo de políticas públicas. Todo lo cual debe generar niveles de confianza que permitan a las personas sentirse parte, a la vez que capturar alguno de los beneficios que se genera y que dan cuenta del capital social. En tal sentido parece importante pensar que a la par del 
crecimiento económico, que es una condición necesaria, también se piense en la distribución de oportunidades.

Se estima que este trabajo realiza un aporte preliminar sobre grandes problemas y desafíos que debe enfrentar la región, cuando se trata de asumir la misión de conducir sapientemente hacia el desarrollo, armonizando y vertebrando adecuadamente confianza, capital social y democracia. Se tiene el convencimiento de que esta triada estratégica puede impeler hacia la apertura de nuevas líneas investigativa, especialmente en una perspectiva de carácter empírico, fuertemente encausado en el desarrollo humano.

Como se puede apreciar en el desarrollo de la investigación, esta deja abierta diversas posibilidades para continuar indagando, las cuales han de ser utilizadas para complementar líneas de trabajo que aporten al fortalecimiento de la confianza como valor social que contribuye al incremento de la calidad de la democracia, lo cual en definitiva, y esta podría ser la hipótesis siguiente, pone a las sociedades en un estadio de desarrollo superior en cuanto a su dinámica social lo que se encuentra más allá de la valoración económica que normalmente se realiza.

- Francisco Ganga Contreras É Professor Titular dol Departamento de Ciências do Desenvolvimento da Universidad de Los Lagos, Chile. E-mail: fganga@ulagos.cl.

Aldo Cassinelli Capurro é Professor Titular da Universidad Central de Chile. E-mail: acassinelli@ucentral.cl.

\section{Referências}

BUROTTO, Juan; GANGA, Francisco. Por una ética de la gestión pública. Revista Polis, v. 11, n. 32, 2012.

CATALÀ, Joan Prats i. Gobernabilidad democrática para el desarrollo humano. Revista Instituciones y Desarrollo, n. 10, p. 103-148, 2001.

CENTRO DE ESTUDIOS PÚBLICOS. Encustas CEP - Desde 1987. 2012. Disponível em: <http://www.cepchile.cl/dms/lang_1/encuestasCEP.htm>. Acesso em: 30 ago. 2012.

FUKUYAMA, Francis. Confianza (trust). Buenos Aires: Atlantida, 1996.

GANGA, Francisco; BUROTTO, Juan Félix. Asimetrías de información entre agente y principal de las universidades chilenas. Revista Estudios Gerenciales, v. 28, n. 122, 2012.

JENSEN, Michael; MECKLING, William. Theory of the Firm: Managerial Behavior, Agency Costs and Ownership Structure. Journal of Financial Economics, v. 3. n. 4, p. 305-360, 1976. 
JORDANA, Jacint. Instituciones y capital social: ¿qué explica qué? Revista Española de Ciencia Politica, n. 2, p. 187-210. 2000.

JUDT, Tony. Algo va mal. Madrid: Taurus, 2010.

KAPSTEIN, Ethan; CONVERSE, Nathan. Por qué fallan las democracias. Journal or Democracy, v. 19, n. 4, p. 160-173, out. 2008.

LATINOBAROMETRO. Corporación Latinobarometro. 2012. Disponível em: <http://www.latinobarometro.org/latino/latinobarometro.jsp>. Acesso em: 10 fev. 2015.

LEVINE, Daniel; MOLINA, José Enrique. La calidad de la democracia en américa latina: una visión comparada. América Latina Hoy, n. 45, p. 17-46, abr. 2007.

LOZARES, Carlos et al. Cohesión, vinculación e integración social en el marco del capital social. Revista Hispana para el Análisis de las Redes Sociales, v. 20. n. 1. p. 1-28, 2011.

MAINWARING, Scott; SCULLY, Timothy. América Latina: ocho lecciones de gobernabilidad. Journal of Democracy, v. 19, n. 3, p. 113-127, jul. 2008.

MONTERO, José Ramón; ZMERLI, Sonja; NEWTON, Ken. Confianza social, confían política y satisfacción con la democracia. Revista Española de Investigaciones Sociológicas, n. 122, p. 11-54, 2008.

MORLINO, Leonardo. Explicar la calidad democrática: ¿qué tan relevante son las tradiciones autoritarias? Revista de Ciencia Politica, v. 27, p. 3-22, 2007.

. Calidad democrática en líderes y partidos. Istituto Italiano di Scienze Humane, Florença, Itália, p. 1-24, 2008. Disponível em: <http://www.ife.org.mx/docs/IFE-v2/CDD/CDDEventosForosAcademicos/EventosForos-2008/PPs-CalidadDemocracia/PPs-CalidadDemocraciadocs/Conferencia01-LeonardoMorlino.pdf>. Acesso em: 15 mar. 2015.

O'DONNELL, Guillermo. Otra institucionalización. La Politica, n. 2, p. 5-28, 1996.

PLASCIENCIA, Jorge Ramírez. Tres visiones sobre capital social: Bourdieu, Coleman y Putnam. Politica y Sociedad, n. 4, p. 21-36, 2005.

PUTNAM, Robert. Bowling alone: America's declining social capital. Journal of Democracy, v. 6, n. 1, p. 65-78, 1995.

VELASCO, Juan Carlos. Deliberación y calidad de la democracia. Revista Claves de la Razón Práctica, n. 167, p. 36-43, 2006.

VILLARROEL, Gladys. Tema con variaciones: el presidencialismo y su impacto en los procesos democráticos. Revista Espacio Abierto, v. 9, n. 1, p. 9-35, 2000.

Texto recebido em 25 de maio de 2015. Aprovado em 03 de agosto de 2015. 